\title{
DEBATE
}

\section{Con: Alzheimer's disease and circadian dysfunction: chicken or egg?}

\author{
Abhay Moghekar and Richard J O’Brien*
}

\begin{abstract}
The development of late-onset Alzheimer's disease is believed to be influenced by genetic, socioeconomic, and lifestyle factors. Recently, converging research in animal and human studies has found that beta-amyloid (A $\beta$ ) levels in cerebrospinal fluid are modulated by sleep-wake cycles. This raises the possibility that chronic sleep loss causes brain amyloid accumulation over time and leads to the development of Alzheimer's disease. The observation that circadian rhythm modulates $A \beta$ levels has not yet been replicated by other groups, and subject selection and methodologies are potential explanations for this. While acute suppression of sleep may raise $A \beta$ levels, it is not known whether chronic sleep loss has the same effect. It is conceivable that altered circadian rhythms are a manifestation of a disrupted sleep network because of preclinical disease, as has been observed in other neurodegenerative disorders. The findings that circadian variation in A $\beta$ levels in cerebrospinal fluid is a direct result of sleep-wake cycles and that altering normal rhythms increases the risk for brain amyloid accumulation need to be replicated in larger cohorts. Prospective studies are needed to decipher whether circadian rhythm dysfunction is a cause, or a result of, amyloid accumulation.
\end{abstract}

\section{Background}

Autosomal-dominant, early-onset Alzheimer's disease (AD) is due to mutations in the processing of the amyloid precursor protein (APP), resulting in excess production of the 42-amino acid form of the beta-amyloid peptide (A $\beta 1-42)$. However, the vast majority of sporadic, lateonset $\mathrm{AD}$ has no similar causative mutations, although some polymorphisms (apolipoprotein E, SORL1, and

*Correspondence: robrien@jhmi.edu

Department of Neurology, Johns Hopkins Bayview Medical Center, Mason F Lord Center Tower, Suite 5100, 5200 Eastern Avenue, Baltimore, MD 21224, USA clusterin) increase the relative risk of this devastating disease [1]. It is believed that late-onset AD develops secondary to a combination of genetic, environmental, and lifestyle factors. Environmental and lifestyle risk factors that have been proposed to increase risk include midlife hypertension, diabetes or impaired insulin resistance, physical activity, obesity, head injury, socioeconomic status, and level of education. Holtzman and colleagues $[2,3]$ have suggested, on the basis of animal and human studies demonstrating diurnal fluctuations of $A \beta$ levels in cerebrospinal fluid (CSF), adding chronic sleep loss in midlife to this mix. This observation has implications not only for $\mathrm{AD}$ pathogenesis but also for the use of CSF for biomarkers in research and clinical management.

\section{Discussion}

Bateman and colleagues [2] first observed the fluctuation of both $A \beta 1-40$ and $A \beta 1-42$ in CSF obtained from research subjects with indwelling spinal catheters, which were sampled hourly for 36 hours. The maximum $A \beta$ levels were two to three times the minimum values for the duration of collection. Using in vivo microdialysis, this same group further demonstrated that, in both wild-type and APP transgenic mice, there was diurnal variation in interstitial fluid (ISF) $A \beta$ levels; peaks occurred during periods of greatest physical activity, and the variation was unrelated to light exposure. Moreover, administration of an orexin antagonist, almorexant, abolished the natural diurnal variation of $A \beta$ whereas orexin administration significantly increased interstitial brain $A \beta$ concentrations [3]. Recently, Huang and colleagues [4], from the same group, replicated the observation of diurnal fluctuation in young healthy subjects but found decreased CSF A $\beta$ fluctuation in subjects with brain amyloid accumulation as ascertained by brain positron emission tomography (PET) scanning with an amyloid-binding agent. These observations are important as they have direct implications for the pathogenesis of $A D$. If $A \beta$ levels in CSF and brain ISF are modulated by sleep-wake activity, then impaired circadian rhythms could cause brain $A \beta$ accumulation and subsequently $A D$.

Although this is certainly a plausible hypothesis, there are some methodological and conceptual issues that need 
to be addressed. In attempts to repeat this important finding, multiple groups looked at diurnal variation of CSF A $\beta$ in humans but could not replicate the findings [5-7]. Although subject selection, the volume of CSF withdrawn, and the interval between collections could account for the lack of fluctuation in some cohorts, $\mathrm{Li}$ and colleagues [5] studied the effect of different intervals between CSF sampling and still did not observe diurnal fluctuation synchronous with sleep-wake cycles. In fact, continuously rising levels of $A \beta$ have been observed in several studies, a finding that lacks a good explanation. Other studies have observed that lumbar $A \beta$ levels are higher than ventricular levels [8,9], although this is controversial [10]. In spite of these controversies, the dynamics of $A \beta$ within the various CSF compartments and its exchange with both ISF and blood $A \beta$ remain important subjects of investigation.

Even if the methodological issues are clarified and the finding of diurnal variation in $\mathrm{A} \beta$ holds true, there is still the concern that these fluctuations are merely associated with neuronal activity in general and are not related to a specific feature of sleep. Cirrito and colleagues [11] demonstrated that $A \beta$ levels are modulated by neuronal activity, and hence it is plausible that these levels fluctuate with sleep-wake cycles. It is well known that, as we age, sleep gets fragmented and as the circadian rhythm desynchronizes this is exaggerated in several neurodegenerative disorders, including Huntington's disease, Parkinson's disease, and AD [12]. This could explain the recent findings of Bateman and colleagues [2] of significantly depressed fluctuations in older normal adults and patients with AD. While sleep abnormalities are frequent in moderate to late $\mathrm{AD}$, it is not known whether these changes play a role in the pathogenesis of the disease. The circadian rhythm, including the maintenance of sleep and wakefulness, involves a complex network with multiple nuclei and neurotransmitter systems. It is conceivable that the normal circadian rhythm is a marker for general integrity of brain networks and that any pathologic process that affects this intricate network causes disruption of circadian patterns. Sleep abnormalities are detected in patients with synucleinopathies (rapid-eye-movement behavior disorder) and schizophrenia, sometimes decades before the manifestation of the disease $[13,14]$. In these disorders at least, it is believed that the sleep dysfunction is just an early preclinical biomarker rather than a cause of the disease. Similarly, it is likely that disrupted circadian patterns could be a preclinical marker for $\mathrm{AD}$. In addition, one might expect a lower prevalence of $\mathrm{AD}$ pathology in patients with narcolepsy, which is characterized by low or absent levels of orexin. Postmortem neuropathologic assessment in a small sample of patients with narcolepsy did not show evidence of a lower prevalence of AD pathology [15].

\section{Summary}

While the data suggesting a causative rather than an associative link between sleep loss and amyloid accumulation are tantalizing, further confirmation of this finding is needed. Prospective cohorts including CSF biomarkers and sleep studies are under way and will help clarify this important observation.

\section{Abbreviations}

$A \beta$, beta-amyloid peptide; AD, Alzheimer's disease; CSF, cerebrospinal fluid; ISF, interstitial fluid.

\section{Competing interests}

The authors declare that they have no competing interests.

Published: 13 August 2012

\section{References}

1. Van Broeckhoven C: The future of genetic research on neurodegeneration. Nat Med 2010, 16:1215-1217.

2. Bateman RJ, Wen G, Morris JC, Holtzman DM: Fluctuations of CSF amyloidbeta levels: implications for a diagnostic and therapeutic biomarker. Neurology 2007, 68:666-669.

3. Kang JE, Lim MM, Bateman RJ, Lee JJ, Smyth LP, Cirrito JR, Fujiki N, Nishino S, Holtzman DM: Amyloid-beta dynamics are regulated by orexin and the sleep-wake cycle. Science 2009, 326:1005-1007.

4. Huang Y, Potter R, Sigurdson W, Santacruz A, Shih S, Ju YE, Kasten T, Morris JC, Mintun M, Duntley S, Bateman RJ: Effects of age and amyloid deposition on A $\beta$ dynamics in the human central nervous system. Arch Neurol 2012, 69:51-58.

5. Li J, Llano DA, Ellis T, Leblond D, Bhathena A, Jhee SS, Ereshefsky L, Lenz R, Waring JF: Effect of human cerebrospinal fluid sampling frequency on amyloid- $\beta$ levels. Alzheimers Dement 2011 Nov 1. [Epub ahead of print].

6. Bjerke M, Portelius E, Minthon L, Wallin A, Anckarsäter H, Anckarsäter R, Andreasen N, Zetterberg $H$, Andreasson U, Blennow K: Confounding factors influencing amyloid beta concentration in cerebrospinal fluid. Int J Alzheimers Dis 2010, 2010. pii: 986310.

7. Moghekar A, Goh J, Li M, Albert M, O'Brien RJ: Cerebrospinal fluid A $\beta$ and tau level fluctuation in an older clinical cohort. Arch Neurol 2012, 69:246-250.

8. Seppälä TT, Nerg O, Koivisto AM, Rummukainen J, Puli L, Zetterberg H, Pyykkö OT, Helisalmi S, Alafuzoff I, Hiltunen M, Jääskeläinen JE, Rinne J, Soininen H, Leinonen V, Herukka SK: CSF biomarkers for Alzheimer disease correlate with cortical brain biopsy findings. Neurology 2012, 78:1568-1575.

9. Talab R, Valis M, Rehak S, Krejsek J: Abnormalities of tau-protein and betaamyloid in brain ventricle cerebrospinal fluid. Neuro Endocrinol Lett 2009, 30:647-651.

10. Tarnaris A, Toma AK, Chapman MD, Petzold A, Keir G, Kitchen ND, Watkins LD: Rostrocaudal dynamics of CSF biomarkers. Neurochem Res 2011, 36:528-532.

11. Cirrito JR, Yamada KA, Finn MB, Sloviter RS, Bales KR, May PC, Schoepp DD, Paul SM, Mennerick S, Holtzman DM: Synaptic activity regulates interstitial fluid amyloid-beta levels in vivo. Neuron 2005, 48:913-922.

12. Lim AS, Saper CB: Sleep, circadian rhythms, and dementia. Ann Neurol 2011, 70:677-679.

13. Boeve BF, Silber MH, Ferman TJ, Lucas JA, Parisi JE: Association of REM sleep behavior disorder and neurodegenerative disease may reflect an underlying synucleinopathy. Mov Disord 2001, 16:622-630.

14. Ruhrmann S, Schultze-Lutter F, Salokangas RK, Heinimaa M, Linszen D, Dingemans P, Birchwood M, Patterson P, Juckel G, Heinz A, Morrison A, Lewis $\mathrm{S}$, von Reventlow HG, Klosterkötter J: Prediction of psychosis in adolescents and young adults at high risk: results from the prospective European prediction of psychosis study. Arch Gen Psychiatry 2010, 67:241-251.

15. Scammell TE, Matheson JK, Honda M, Thannickal TC, Siegel JM: Coexistence of narcolepsy and Alzheimer's disease. Neurobiol Aging 2012, 33:1318-1319.

\section{doi:10.1186/alzrt129}

Cite this article as: Moghekar A, O'Brien RJ: Con: Alzheimer's disease and circadian dysfunction: chicken or egg? Alzheimer's Research \& Therapy 2012, $4: 26$. 